\title{
Detection of Human Facial Expression using CNN Model
}

\author{
Srilakshmi Ch, Kiruthika K, Bharathi Priya R, Jayalakshmi J
}

\begin{abstract}
Facial expression is the most effective and herbal non verbal emotional conversation method People can range indoors the way they display their expressions Even pics of the same character within the identical countenance can vary in brightness historical past and pose and these variations are emphasized if thinking about particular subjects because of versions in shape ethnicity amongst others Hence countenance recognition remains a challenging trouble in PC vision To advise a solution for expression reputation that uses a combination of Convolutional Neural Network and precise picture prepossessing steps It defined the modern-day solution that has green facial capabilities and deep gaining knowledge of with convolutional neural networks CNN's has achieved high-quality success within the classification of assorted face emotions like glad angry unhappy and impartial Hundreds of neuron smart and layer smart visualization techniques have been applied the usage of a CNN informed with a publicly to be had photo data set So it's positioned that neural networks can capture the colors and textures of lesions unique to respective emotion upon analysis which resembles human desire making.
\end{abstract}

Keywords: Face Expression, Deep learning, Tensor flow.

\section{INTRODUCTION}

$\mathrm{F}$ acial expressions are one in every of the more critical elements of human communication. The face is chargeable for communicating now not only mind or ideas, however also feelings. Facial bio metrics are regularly being carried out to extra industries, disrupting design, manufacturing, construction, enforcement, and health care.Many strategies are proposed to find out human faces in images, they will be classicfied into four categories: knowledge-based techniques, feature-based methods, template-primarily primarily based strategies, and appearance-based totally strategies. When used separately, those methods can't resolve all of the troubles of face detection like pose, expression, orientation, and occlusion. Hence, it's better to paint with numerous successive or parallel techniques. Most of the reputation techniques, facial skills reported to this point are centered on the popularity of six primary expression categories which include emotions like sad, anger, happy, disgust, fear and anger.

Revised Manuscript Received on June 10, 2020.

* Correspondence Author

Ms Srilakshmi Ch*, Assistant Professor, Department of Information Technology, R. M. D Engineering College, Thiruvallur, Tamilnadu, India. E-mail : sri2222lakshmi@gmail.com

Kiruthika K, Student, Department of Information Technology, R. M. D Engineering College, Thiruvallur, Tamilnadu, India. E-mail: krkiruthika02@gmail.com

Bharathi Priya R, Student, Department of Information Technology, R. M. D Engineering College, Thiruvallur, Tamilnadu, India.

Jayalakshmi J, Student, Department of Information Technology, R. M. D Engineering College, Thiruvallur, Tamilnadu, India.

(C) The Authors. Published by Blue Eyes Intelligence Engineering and Sciences Publication (BEIESP). This is an open access article under the CC BY-NC-ND license (http://creativecommons.org/licenses/by-nc-nd/4.0/)

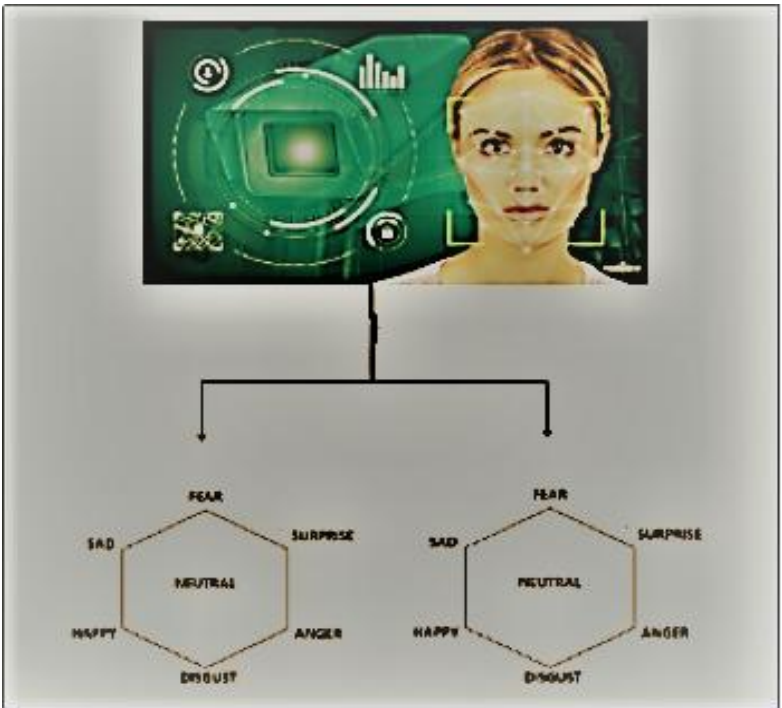

Fig: SFEH(Six Facial Expressions Hexagon) Model

A face is the seen manifestation of the powerful kingdom, cognitive activity, intention, character, and psychopathology of a personal and performs a communicative function in interpersonal relations. Facial expressions are related to the six universal feelings, particularly Joy, Surprise, Disgust, Sadness, Anger, Fear, moreover as Neutral. Our facial emotions are expressed through the activation of specific devices of facial muscles. These sometimes subtle, yet complicated alerts in expression frequently incorporate an abundant quantity of records about our united states of the mind.

Automatic Recognition of Facial Displays of Knelt Emotions. Humans regulate their facial expressions to speak their internal states and every now and then to lie to observers concerning their real emotional states. Evidence in psychological technology shows that discrimination facial responses are brief and subtle. It may even be hired in behavioral technological knowledge and scientific practice. It's been studied for an extended period of a while and acquired progress in recent decades. Though a good buy of development has been made, recognizing facial capabilities with an excessive accuracy stays to be tough thanks to the complexity and types of facial expressions.

On a day to day basis humans typically recognize feelings by using feature features, displayed as an issue of facial features. For instance happiness is undeniably associated with a grin or an upward motion of the corners of the lips. Similarly different emotions are characterized through means of various deformations present day to a particular expression. Research into automatic popularity of facial expressions addresses the issues surrounding the example and categorization of static or dynamic characteristics of those deformations of face pigmentation.

Published By:

Blue Eyes Intelligence Engineering

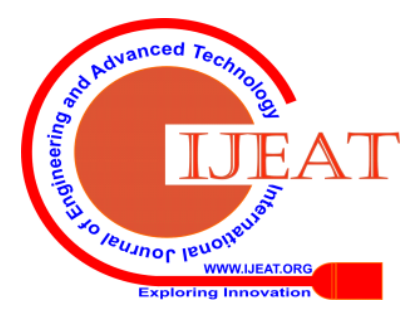




\section{RELATED WORK}

Appearance Identity Fusion Network for Facial Expression Recognition handled by Haifeng Zhang, Wen Su4 and Zengfu Wang in the year 2019.As a critical subfield of face acknowledgment, outward appearance acknowledgment has drawn expanding consideration from the PC vision network. It makes a wide cluster of utilizations extending from exhaustion reconnaissance, separation learning, human PC cooperation to clinical treatment.It watched a demeanor personality combination system to address the incredible between subject varieties in outward appearance acknowledgment. The model is intended to together learn personality related highlights and articulation related highlights by means of two branches with a similar articulation picture input. A bilinear module is acquainted with intertwine two sorts of highlights and become familiar with the connection between them.Our technique beats a large portion of the cutting edge. Our strategy accomplishes 96.02\% and 85.21\% acknowledgment precision, separately. Character related highlights and articulation related highlights are melded and the connection between them is found out.

Face Expression Recognition Based on Deep Convolution Network prepared by Minjun Wang, Zhihui Wang and Shaohui Zhang in year of 2018.It utilized profound convolutional neural system model in profound figuring out how to extricate facial highlights and uses Soft max classifier to perform outward appearance characterization. The calculation utilized in this paper doesn't require human investment in guided learning, and gives a computerized include extraction technique, with the goal that the distinguished impact is better. It did investigates JAFFE and $\mathrm{CK}+$ database and contrasts it and different techniques. The test results show that this strategy is for sure more viable than other hand-extraction facial component acknowledgment strategies. This paper utilizes jaffe and $\mathrm{ck}^{+}$two face appearance libraries to confirm the calculation, demonstrates the viability of the calculation, and shows that its exhibition is superior to the conventional technique.

Outward appearance Analysis for Distress Detection handled by Priyanka Nair and Subha V in the time of 2018. We can recognize the individual we are taking a gander at and give data like age, sexual orientation, engaging quality and numerous other comparative subtleties. While having a discussion people normally incorporate articulations as a piece of their discourse to deduce the genuine expectation of the individual. Outward appearances are a consequence of developments of little facial muscles (particularly those around eyes, cheeks, mouth corners and so on.). The face and facial tourist spots discovery are finished utilizing Viola Jones calculation. Facial patches dynamic during a feeling elicitation are then removed for surface examination. The component extraction technique utilized here is Gray Level Difference Method (GLDM) in which surface highlights are gotten from the GLDM likelihood thickness capacities. The subsequent stage, characterization is finished utilizing Naïve Bayes Classifier. Concerning the prepared data, the feeling of the individual is perceived and is utilized for deciding his trouble level and the tried outcome utilizing Extended Cohn Kanade(CK+) and Japanese female outward appearance (JAFFE) datasets. It watched techniques for outward appearance acknowledgment utilizing GLDM and consequently utilizing this acknowledgment result to decide if the individual is upset or not. Face and facial tourist spots are recognized from the information picture and afterward facial patches are separated to which GLDM is applied to determine the element vector. These element vectors are grouped utilizing Naïve Bayes classifier and consequently trouble investigation is finished.

\section{THEORETICAL BACKGROUND}

To come across the face expression it planned to layout a deep mastering technique so that someone with lesser facts in software needs to also be able to use it easily. It proposed a gadget for predicting facial expression. It explains the experimental assessment of our methodology. Samples of a greater wide range of photographs are amassed that include different education together with happy, angry, sad, etc. Different variety of pix is amassed for each elegance that emerges as labeled into database snapshots and enters pics. The primary attributes of the picture have relied upon the shape and texture orientated features.

The recent fulfillment of convolutional neural networks (CNNs) in duties like item elegance extends to the trouble of countenance recognition. Within the subsequent sections, we're going to present a definition of our hassle to classify pictures of human faces into discrete emotion categories. Many hooked up countenance recognition (FER) structures use standard gadget mastering and extracted features, which no longer have huge performance when accomplished to previously unseen data. We implemented 3 extraordinary classifiers from scratch:

- A baseline classifier with one convolutional layer

- A CNN with a difficult and rapid duration of five convolutional layers

- A deeper CNN with a parameter range of convolutional layers, clear out dimensions, and quantity of filters. For every one of those models, we tuned parameters including mastering rate, regularization, and dropout.

We also experimented with the usage of batch normalization and fractional max-pooling and implemented more than one classifier the usage of fine-tuning with variations on the number of layers retained, the number of layers once more propagated through, and additionally, the initial network used.

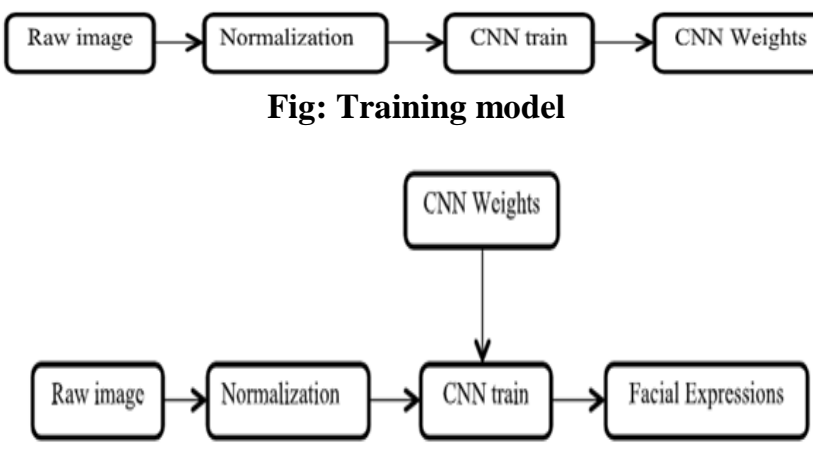

Fig: Testing model

\section{METHODOLOGY}

The facial expression recognition device is implemented in the use of convolutional neural networks. During training, the machine receives training data comprising greyscale pics of faces with their respective expression label and learns a collection of weights for the community. The schooling step was to enter a picture with a face. Thereafter, intensity normalization is carried out to the image. The normalized photos are wont to teach the Convolutional Network. To verify that the schooling performance isn't always plagued with the resource of the order of presentation of the examples, a validation dataset is employed to determine on the final excellent set of weights out of a group of educational physical activities finished with samples furnished in numerous orders.

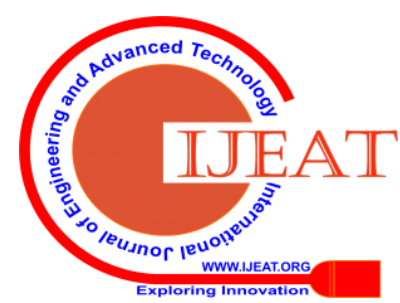


The output of the training step may want to be a fixed of weights that gain the handiest end result with the education information. During the take a look at, the device obtained a grayscale picture of a face from the take a look at the dataset, and output the predicted expression by manner of the usage of the closing community weights determined out at some stage in education. Its output may be a single variety that represents one of the various seven number one expressions.

\section{A. CNN Model Steps:}

I. Conv2D: It is the layer to sophisticate the images into multiple pics is the activation feature.

II. MaxPooling2D: It is used to combine the rate from the given size matrix and same is used.

III. Flatten: It is used to flatten the size of the photo acquired after convolving it.

IV. Dense: It is used to make this a completely connected version and is the hidden layer. $\mathrm{V}$

V. Dropout: It is used to keep away from overfitting on the dataset and densely the output layer contains at best one neuron which makes a decision which category picture belongs.

VI. Image Data Generator: It is that resizes the image, applies shear in some range, zooms the photo, and does horizontal flipping with the picture. This Image Data Generator consists of all viable orientation of the photo.

VII. Training Process: train datagen. Test datagen. Flow from directory is used to prepare to test facts for the version and all are just like above.

VIII. Epochs: It tells us the quantity of instances the version may be skilled inside the forward and backward pass.

IX. Validation process: validation data is used to feed the validation test facts into the model. Validation steps denote a wide variety of validation and take a look at samples.

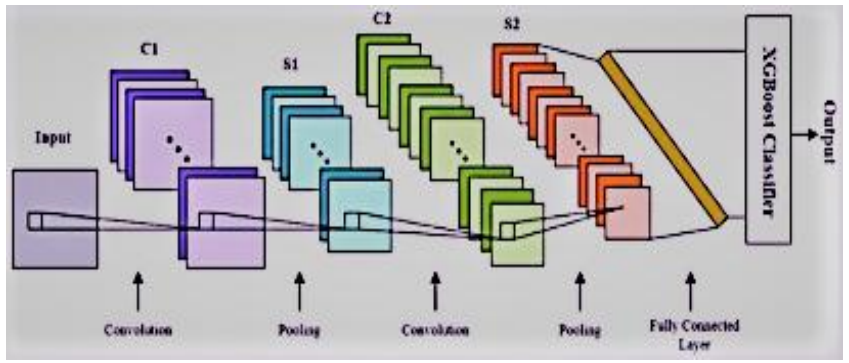

Fig. CNN Model

\section{B. Architecture of $\mathrm{CNN}$ :}

A common architecture of a convolutional neural community consists of An input layer, some convolutional layers, a few fully-related layers, and an output layer. CNN is designed with a few modifications to LeNet Architecture. It has 6 layers without considering enter and output.

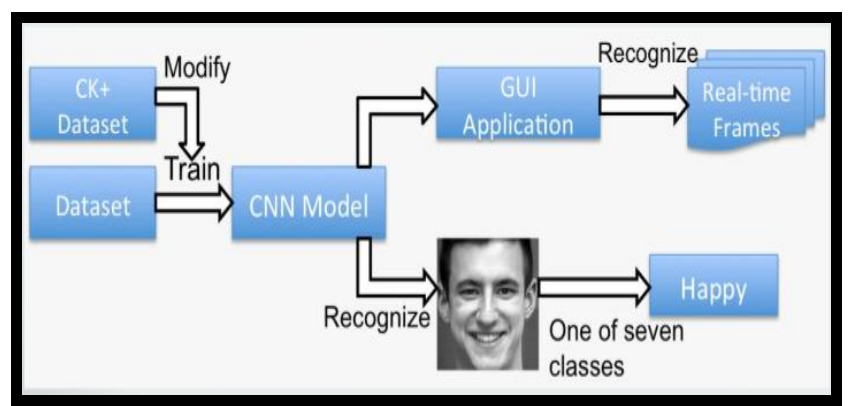

Fig. Architecture of CNN
1) Input Layer: The input layer has predetermined, constant dimensions, so the image wants to be processed before it is able to be fed into the layer. Normalized grayscale snap shots of length forty eight X 48 pixels from a given dataset are used for education, validation, and trying out. For attempting out proposed laptop webcam pixels are also used, in which the face is cropped and detected using the OpenCV in Haar Cascade Classifier and normalized.

2)Convolution and Pooling (ConvPool) Layers: Convolution and pooling are performed primarily based on batch processing. Each batch has $\mathrm{N}$ photographs and $\mathrm{CNN}$ filter out weights are updated at the one's batches. Each convolution layer takes a photo batch center of 4 duration $\mathrm{N} \times$ Color-Channel $\mathrm{x}$ width $\mathrm{x}$ pinnacle. Feature map or clear out for convolution is also 4 dimensional (Number of feature maps in, a wide variety of function maps out, filter width, clear out the pinnacle). In every convolution layer, four-dimensional convolutions are calculated among photo batch and function maps. After convolution is exceptional the parameter that changes is photograph width and peak.

- New image width $=$ old image width - clear out width +1

- New picture peak $=$ old photograph peak - easy out top + 1

In this venture max pooling is finished after convolution. Pool length of (2x2) is 12 taken, which splits the picture right into a grid of blocks each of length 2x2 and takes the most of four pixels. After pooling, the first-rate peak and width are affected. Two convolution layers and a pooling layer are used inside the structure. The first convolution layer size of the input image batch is $\mathrm{Nx} 1 \mathrm{x} 48 \mathrm{x} 48$. Here, the dimensions of the photo batch is $\mathrm{N}$, the wide range of coloration channels is 1 and both photo pinnacle and width are forty eight pixels. Convolution with a function map of $1 \times 20 \times 5 \times 5$ results photograph batch is of size $\mathrm{Nx} 20 \times 44 \times 44$. After convolution, pooling is finished with a pool duration of $2 \times 2$, which results in a photo batch of duration Nx20x22x22. This is observed with the beneficial aid of a $2 \mathrm{~d}$ convolution layer with a feature map of $20 \times 20 \times 5 \times 5$, which ends up in a photograph batch of duration Nx20x18x18. This is accompanied by utilising a pooling layer with pool duration $2 \times 2$, which leads to a photograph batch of length Nx20x9x9.

3) Fully Connected Layer: This layer is inspired through the way alerts are transmitted to the brain through neurons. It takes an outsized form of input features and transforms skills thru layers related with trainable weights. Two hidden layers of duration 500 and 300 gadgets are implemented in fully-associated layers. The weights of these layers are trained to make use of forwarding propagation of coaching facts then backward propagation of its errors. Backpropagation starts off evolving from evaluating the difference among prediction and authentic value and once more calculates the burden adjustment wished to each layer before.

We can control the education velocity and consequently the complexity of the structure via tuning the hyper-parameters, like studying fee and community density. Hyperparameters for this deposit consist of learning fee, momentum, regularization parameter, and decay. The output from the second pooling layer is of duration Nx20x9x9 and the input of the number one hidden layer of the fully-connected layer is of period $\mathrm{Nx} 500$. So, the output of the pooling layer is flattened to Nx1620 period and fed to the number one hidden layer. The output from the number one hidden layer is fed to the second one hidden layer. The 2nd hidden layer is of period Nx300 and its output is fed to the output layer of a length adequate to the quantity of facial capabilities classes.

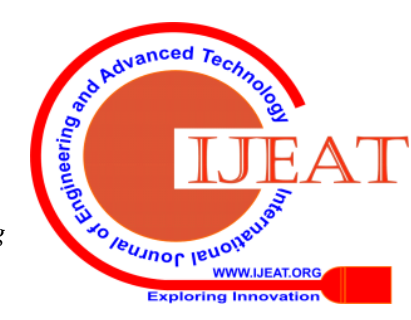




\section{Detection of Human Facial Expression using CNN Model}

Four Output Layer: Output from the second one hidden layer is connected to the output layer having seven distinct education and output is obtained using the opportunities for each one of the seven instructions. The magnificence with the highest possibility is the predicted elegance.

\section{PROPOSED WORK}

This section details the data used for training and testing, how the data was preprocessed, the various models that were used, and an evaluation of each model.

\section{MODULES:}

- Detection of human face recognition (Module-01)

- To capture the face expressions (Module-02)

- To train a given dataset by CNN model (Module-03)

- Classification of human facial expression through the given input image (Module -04)

- Classification of human facial expression through real-time video captures (Module-05)

\section{A. Module-01: Detection of human face recognition}

Ongoing face discovery includes the recognition of a face from a grouping of edges from a video taking pics gadget. While the equipment necessities for such a framework are a long way increasingly rigid, from a PC vision angle, ongoing face identification is a far easier gadget than distinguishing a face in a static photo.

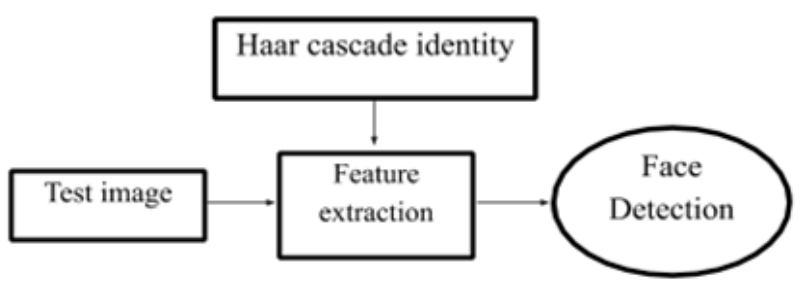

Fig. Detection of Human face

Pre-requisites:

- Opencv installed

- A running webcam

Open Source Computer Vision is abbreviated as OSCV which is a pc imaginative and prescient library that consists of various abilities to perform operations on Images or videos. Opencv library may be used to perform a couple of operations on videos. It comes with many powerful video enhancing functions. In cutting-edge scenarios, techniques such as photograph scanning, face recognition can be accomplished using OpenCV. This library can be used to carry out a couple of operations on videos. Let's try to do some interesting component usage of cv2 or cv based completely on the python version package. Take a video as input by webcam and wreck the video into a frame by way of the method of body and store that body. Now, some of the operations can be completed on those frames like reversing the video report or crop the video, etc.

\section{B. Module-02: To capture the face expressions}

Step by using step manner to seize the given image:

- To import the fundamental libraries.

- Then 'cv2.VideoCapture(zero)' begins catching from the default camera(here zero methods default camera).
- Capturing outlines in an endless circle will be the accompanying advance. From that point forward, it changes over the caught video into a dark scale through 'cv2.COLOR_BGR2GRAY' command(Open - CV catches in Blue-Green-Red rather than Red-GreenBlue).

- We yield each frame(original video and dim scaled video) with the guide of 'cv2. I appear' order

- To stop the arrangement, we need to layout a key. For our situation "s" is the key we have characterized to intrude on the circle.

- Lastly we discharge the entirety of the cameras from the open-CV and demolish the entirety of the home windows opened out of sight. This is done in the event that you need to discharge the entirety of the foundation strategies happening subsequent to stopping the arrangement.

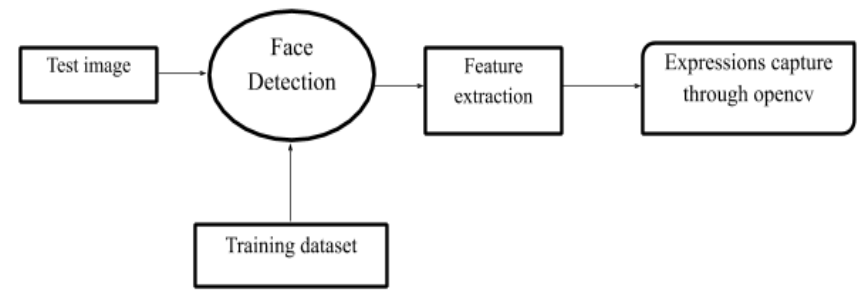

Fig. Detection of Human facial Expression

Gray Scale Base: Gray information within a face also can be dealt with as crucial features. Facial capabilities which include eyebrows, pupils, and lips appear usually darker than their surrounding facial regions. Various recent feature extraction algorithms look for nearby grey minima inside segmented facial regions. The input photos are enhanced by assessment-stretching and grey-scale morphological routines in order to improve the high-quality of local dark patches and to make detection easier. The extraction of darkish patches is performed by a low-level gray-scale threshold. Based method and consist of three levels. Faces greyscale behavior in pyramid photographs and it utilizes hierarchical Face location steady three levels. Higher two levels based totally on pix at distinct resolutions and the lower level, part detection approach.

Edge Base: This work was based totally on studying line drawings of the faces from photographs, aiming to find facial functions. Initially the pics are enhanced with the aid of making use of median filter for noise removal and histogram equalization for evaluation adjustment.

\section{Module-03: To train a given dataset by CNN model}

Neural networks are gaining a good deal extra attention in lots of pattern popularity problems, item recognition, and autonomous robotic driving. Since face detection with the splendor pattern recognition problem, numerous neural community algorithms were proposed. The benefit of the usage of neural networks for face detection is the feasibility of training a device to capture the complicated elegance conditional density of face patterns. However, one demerit is that the network architecture has to be notably tuned (quantity of layers, a wide kind of nodes, analyzing rates, etc.) to get more performance. The first degree having parallel subnetworks in which the inputs are filtered intensity values from an original picture. The inputs to the second one-degree community encompass the outputs from the sub-networks and extracted characteristic values. 
Output at the second one degree indicates the presence of a face inside the input region. Their network is composed of four layers with 1,024 enter devices, 256 devices in the first hidden layer, eight devices within the second hidden layer, and output devices.

Libraries Required:

Open CV:

Opencv (Open Source Computer Vision Library) is an open source PC imaginative and prescient and device getting to know software library. OpenCV finally ends up constructed to offer a common infrastructure for laptop imaginative and prescient programs and to reinforce the usage of system perception in enterprise products. Being a BSD-licensed product, Opencv makes it clean for corporations to make use of and alter the code. The library has more than 2500 optimized algorithms, which includes an entire set of each conventional and state-of-the-art laptop vision and gadget learning algorithms.

\section{Numpy:}

NumPy enriches the programming language Python with effective facts systems for inexperienced computation of multi-dimensional arrays and matrices. Besides that the module property a large library of excessive-degree mathematical skills to feature on these matrices and arrays. It is the crucial package deal deal for clinical computing with Python. It includes numerous abilities which encompass those important ones.

SciPy: SciPy (Scientific Python) is often said within the same breath as NumPy. Numeric is like NumPy, a Python module for high-performance, numeric computing, however, it's miles out of date nowadays.

Tensor Flow: It is a basic library that can be used to create Deep Learning fashions at once or through the usage of wrapper libraries that simplify the device constructed on the pinnacle of Tensor Flow.

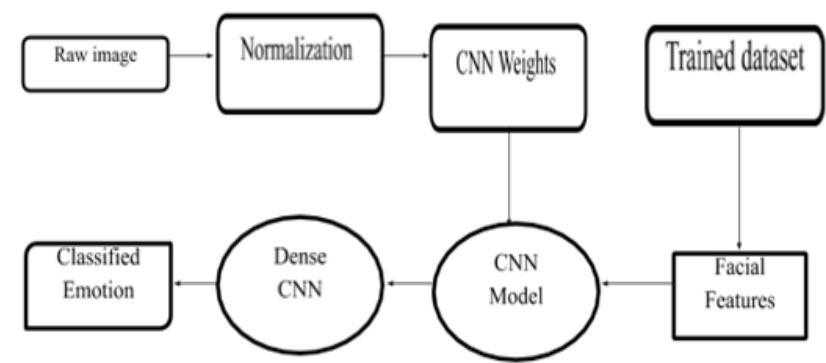

Fig. Training a Dataset of the Model

\section{Module-04: Classification of human facial expression through the given input image}

The facial features recognition machine is implemented using convolutional neural networks. CNN structure to predict simple expressions from facial components is proposed and it combines standard strategies for face and facial areas detection/extraction and convolutional neural community for function extraction and classification. The fundamental contributions are:

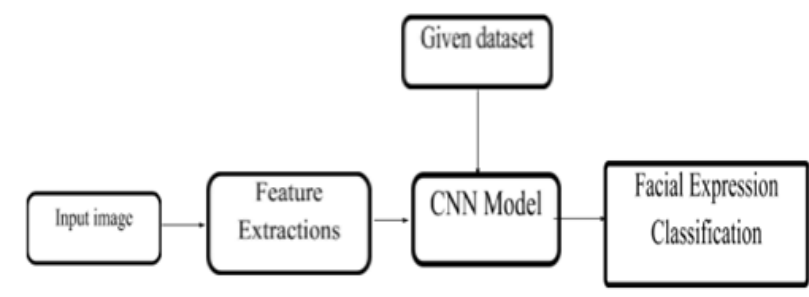

Fig. Facial Expression of given input image

Using facial parts as inputs to lessen the scale of enter and FER processing time when in comparison to different CNN architectures that used the entire face for facial features recognition. Proposing two-channel CNN architecture for characteristic extraction and expression popularity. The facial features reputation technique is based totally on a two-channel architecture that is in a position to apprehend facial expressions. The detected facial components are cropped and extracted and then used as the input into the inception layer of the CNN. The Training phase includes the characteristic extraction and types of the usage of convolution neural networks.

\section{E. Module-05: Classification of human facial expression through real-time video captures}

For outward appearance acknowledgment issues, the acknowledgment technique is isolated into two stages, one is face portrayal, and other one is classifier development. In the face portrayal step, highlights identified with outward appearance are taken from pictures. A portion of the capacities are hand-planned, though others are found out from tutoring pictures. At that point, the dimensionality of the highlights is diminished to encourage a proficient kind and decorate the speculation usefulness and the standard articulations which are refered to in normally outrage, appall, dread, euphoria (or joy), bitterness, and shock, while a few scientists include unbiased as the seventh articulation. The classifiers are structured based absolutely at the disentangled capacities to group each articulation as one of the six (or seven) articulations. Notwithstanding, Convolutional Neural Network (CNN), a sort of profound learning approach, can take in highlights from training information. CNN learns works through a blend of convolutional layers and subsampling layers, and regularly followed by a rigid of totally related layers. CNN is getting progressively well known in the most recent years as a result of its green execution.

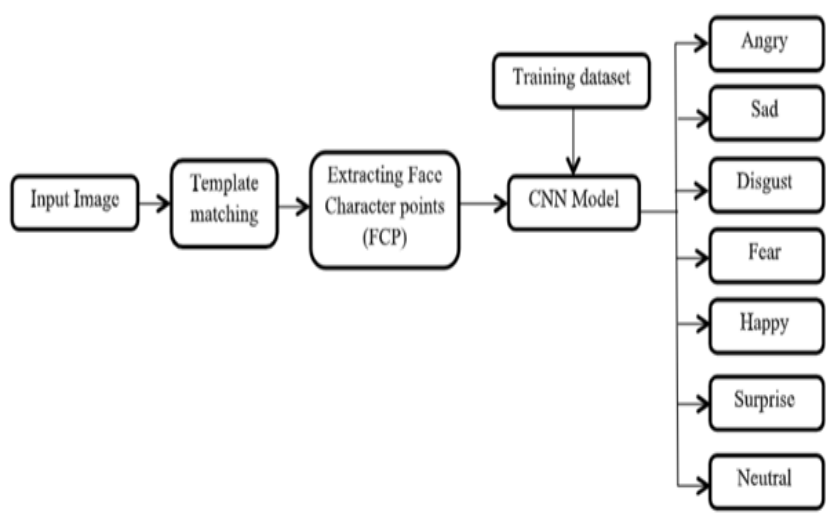

Fig. Classification of Human facial Expression

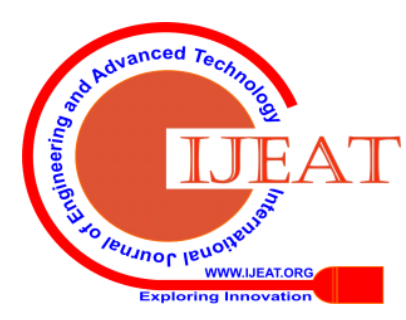




\section{Detection of Human Facial Expression using CNN Model}

\section{RESULT}

The objective of this project is to categorize human faces into one among the six universal emotions or a 7th neutral emotion. In current years, many papers are posted that use deep getting to know for facial emotion recognition. These papers used freely to have datasets with the kingdom of the art models achieving an accuracy of 0.66 . With this in mind, a lot of various models each new and old had been experimented with to make a final model with comparable results.

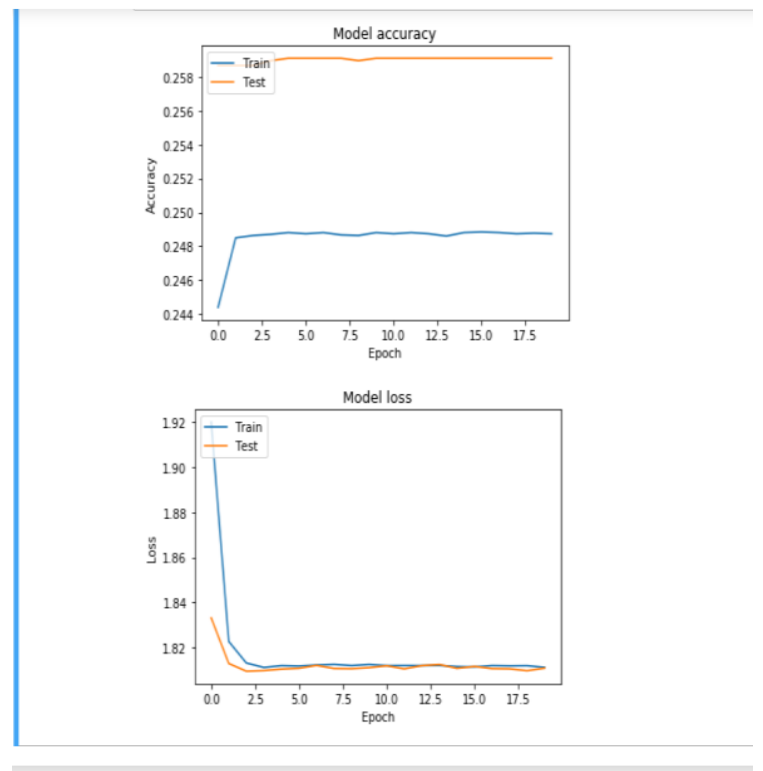

Fig. Accuracy of the datasets
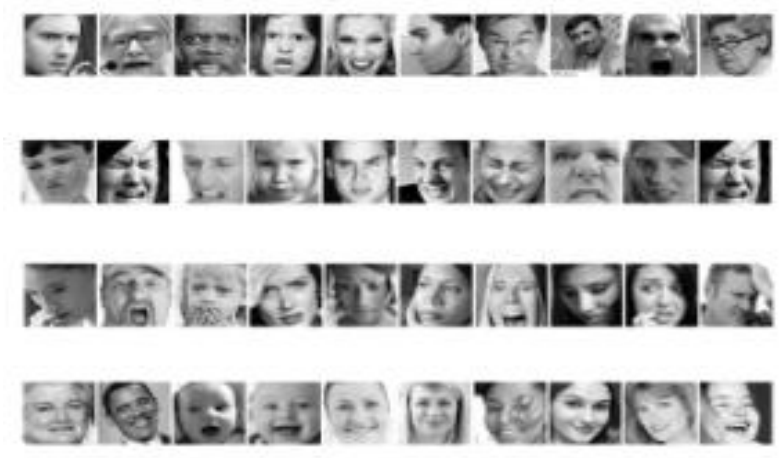

\section{DFत्ञ}

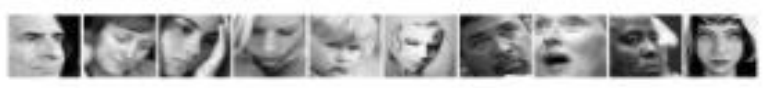

Fig. Different facial expression data sets given train the algorithm.

\section{FUTURE ENHANCEMENT}

To extend our model to paint pictures. This may enable the U.S. to analyze the effectuality of pre-trained models for facial feeling recognition. To automate this method by showing the prediction end in an internet application or desktop application and to optimize the work to implement in synthetic Intelligence surroundings.

\section{CONCLUSION}

In this paper, the aim was to classify facial expressions into one among seven emotions by mistreatment varied on the FER2013 dataset. Models that were experimented with embody call trees, feed-forward neural networks, and smaller convolutional networks before inbounding at the planned model. It classified the image of faces in which any of 7 separate emotion classes that shows universal living being human emotions and experimented with varied techniques, cherish fine-tuning, and halfway max-pooling.

\section{REFERENCES}

1. Constants across societies inside the face and feeling. Diary of disposition and brain science, P. Ekman, and W. V. Friesen. 17(2), 124-129. (1971)

2. http://www.kaggle.com/c/challenges-inrepresentationlearning-facial-ex pression-recognition-challenge (2013)Challenges in illustration learning: face expression recognition challenge.

3. face expression Recognition with Convolutional Neural Networks. CS231n Course comes. Raghuvanshi, A., \& Choksi, V. (2016).

4. Static Facial Expressions in powerful Conditions: knowledge, analysis Protocol And Benchmark, IEEE International Workshop on Benchmarking Facial Image Analysis Technologies gibe, IEEE International Conference on pc Vision ICCV2011, Barcelona, Spain, 6-13 Gregorian calendar month 2011 ,Abhinav Dhall, Roland Goecke, Simon Lucey, and Tom Gedeon. (2011)

5. grouping giant, Richly Annotated Facial-Expression Databases from Movies, IEEE transmission, 19(3):3441, July 2012 Abhinav Dhall, Roland Goecke, Simon Lucey, Tom Gedeon. (2012).

6. Q. V. Le, N. Jaitly, and G. E. Hinton. (2015). a direct gratitude to introducing enduring systems of rectified straight units. arXiv preprint arXiv:1504.00941.

7. (2016) IEEE Winter Conference on Applications of pc Vision (WACV), Lake Placid, NY, 1-10. A. Mollahosseini, D. Chan, and M. H. Mahoor. (2016).

8. face expression Recognition with Identity and feeling Joint Learning. IEEE Transactions on emotional Computing. 1-1.Li M., Xu H., Huang X., Song Z., Liu X. and Li X. (2018).

\section{AUTHORS PROFILE}

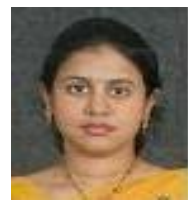

Ms.Srilakshmi completed M.E Computer Science from Anna University and pursuing Ph.D.in Anna University Chennai, she has published several papers concerning data mining, image processing, big data analytics, deep learning, machine learning and wireless networks. Currently she is working as assistant professor in R.M.D Engineering College, Tamil Nadu.

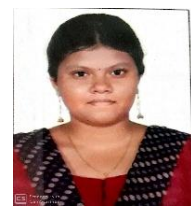

Kiruthika K, currently pursuing a bachelor's degree in the stream of Information Technology at R.M.D Engineering College, Thiruvallur, TamilNadu, India.She is interested in the fields of Cyber security, App development and website development.She has done several projects related to application development.

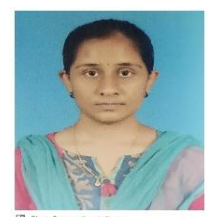

Bharathi Priya $\mathbf{R}$, currently pursuing a Bachelors's degree in the stream of Information Technology in RMD Engineering College, Thiruvallur, Tamilnadu, India.She has experience in using Amazon Web Services and spring MVC and worked on projects related to that.she is interested in the front end web development ,Data visualisation and completed Data Camp certification courses on python for data visualization and basics of machine learning,also completed NPTEL certification course on python and c programming.

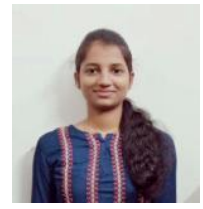

Jayalakshmi J, currently pursuing a Bachelors's degree in the stream of Information Technology in RMD Engineering College, Thiruvallur, Tamilnadu, India.She has experience in full-stack development and worked on projects in that.She is interested in the fields of Cloud Computing, Web development and completed NPTEL certification courses on JAVA, Cloud Computing and C

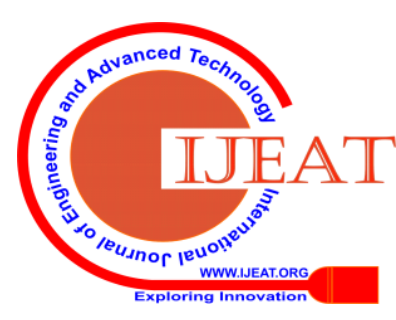

